\section{THE MEANING OF TACHYCARDIA.}

BY R. MCNAIR WILSON, M.B., CH.B. GLASG. (Read before the Fellowship of Medicine.)

AFTER the recent influenza epidemic a number of cases presented themselves in which a rapid pulserate appeared to be the chief symptom. The clinical picture was that of convalescence, a rather slow convalescence. The patients tended to be breathless and occasionally complained of feeling giddy. If their attention had been called to their pulses they usually showed anxiety at the fast rate. On the other hand, they were often quite unaware that their pulses were quick at all. Many of them were apparently well and capable of considerable exertion. Other cases of tachycardia have been met with in patients suffering from dysentery, chronic malaria, chronic trench fever, and other diseases.

The chief physical sign is the quick pulse, which may reach 120 or higher, but which is commonly between 90 and 110. This pulse quickens still further on exertion, running up often to 160, as recorded by the polygraph. Moreover, there is a normal "release" with atropine which, when given in doses of $1 / 33 \mathrm{gr}$. subcutaneously, produces an acceleration of $20-25$ beats a minute.

This normal release led to the view that the vagus nerve in these cases is normal in all respects, but evidence gradually accumulated which pointed to a different conclusion. It was found that if the patients were instructed to take a long deep breath and hold it marked slowing of the pulse (Fig. 1) or a marked loss of pulse volume with quickening was wont to occur. After atropine this phenomenon could not be elicited. (Fig. 2.) Moreover, in a series of controls who had not recently suffered from any acute illness and who were not the victims of "rheumatism" no slowing and no loss of volume occurred. It was concluded that the phenomena were abnormal, and that they depended on the vagus for their production.

The next question is: The vagus is not less excitable than normal in these cases; is it more excitable than normal? It was argued that there are two functions of the heart which are distinct, augmentation and acceleration. Augmentation is accomplished when a more prolonged or larger diastole is followed by a larger systole. Both cardiac nerves, the vagus and the sympathetic, take part in this mechanism, the vagus by increasing the length of diastole, the sympathetic by responding in systole. Acceleration, on the contrary, is a purely sympathetic phenomenon. Now the effect of atropine is, for the moment, to inhibit the vagus, and so to cause the nervous mechanism of augmentation to break down. Thus acceleration alone remains if the circulation is to be maintained. This is the so-called "release of the pulse" under atropine. If, however, atropine were to be given in a dose small enough not to inhibit the vagus but somewhat to lower its excitability, we should discover by the result whether or not the nerve was hyper-excitable in those cases of tachycardia. For if the tachycardia should be due to an excessive vagus pull com. pensated by acceleration the act of lowering the excitability of the vagus to normal must cause the pulse to slow down; compensatory acceleration would no longer be necessary.

Four cases with pulse-rates at rest, varying from 108 to 85, were selected; all these showed tachycardia when not at rest, and one control who was healthy was added to the number. A dose of $1 / 2 \mathrm{CO}$ gr. atropine was given intravenously. The results are shown in Fig. 3 .

It was then seen that a very small dose of atropine was capable, in these cases of tachycardia, of slowing the pulse markedly. In the control case (b), on the contrary, quickening took place. Clearly, then, a slight lowering of vagus excitability produced slowing, or, in other words, the accelera. tion was compensatory to excessive vagus action. Numerous other experiments on the same lines were carried out with the same results.

\section{Function of the Vagus.}

It appeared that the conception of the vagus, which was expressed by Luciani, the Italian physiologist, as the "nerve of diastole," was justified. For this nerve would not seem to be inhibitor in the narrow sense, but rather to act by increasing the filling time of the heart in response, no doubt, to stimula. tion from the cortex. A vagus "pull" thus initiates a period of increased cardiac effort, what is spoken of as increased "output per beat." But a prolonged diastole with consequent increased filling of the heart presupposes a more powerful systole. In other words, the whole level of cardiac activity is raised. If the heart, on the other hand, should not be able to make this response in each individual beat it will require to add acceleration to augmentation-it will require, that is to say, to increase output per minute, since output per beat is not sufficient.

In cases where the vagus is hyper-excitable there will always be a tendency every time that influ. ences pass from the cortex to the medullary centres -i.e., on excitement or calls for effort-for the vagus to overdo its part and to prolong diastole unduly. The result must be a compensatory acceleration if the necessary level of activity is to be maintained. Professor F. A. Bainbridge, dealing with the purely muscular question, has recently written :-

"In response to a moderate increase in venous inflow a comparatively slight dilatation enables a powerful (thick-walled) ventricle to increase considerably its out put per beat, and still to empty itself almost completely at each beat. Hence a slight increase in the pulse-rate will bring about a sufficiently large output per minute to prevent any accumulation of blood on the venous side, and therefore to prevent an appreciable rise of venous pressure. Placed under the same conditions a feeble (thin-walled) ventricle, even when dilated to its physiological limit, may be unable greatly to increase its output per beat and may be far from empty at the end of systole. In these circumstances considerable acceleration of the pulse will be necessary to increase the minute volume of the heart sufficiently to balance the venous inflow. In this way acceleration of the pulse can compensate for lessened contractile power in maintaining the output of the heart per minute."

The work described above suggests that an over. active vagus imposes a similar strain on a heart not necessarily weakened at all. But there is another aspect of the question, which must now be introduced. During the work on tachycardia a case was encountered, tracings from which, and from another similar case, are shown. (Figs. 4 and 5.)

In this case on deep inspiration there was a preliminary slowing from 95 to 75 . And then quite suddenly the blood pressure, which had been $120 \mathrm{~mm}$. Hg systolic, fell to 80 systolic, the pulse ran up to 100 and later to 110 , and the patient felt faint. Thus both slowing and loss of volume were seen in the same tracing. It appeared that 
FIG. 1.

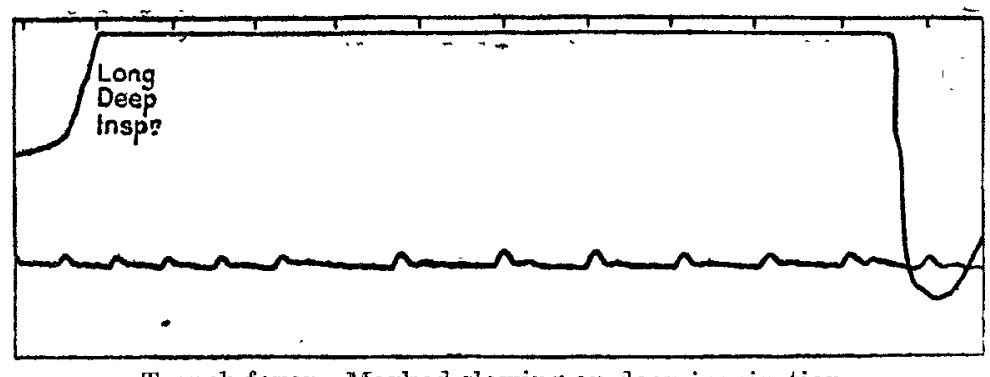

Trench fever. Marked slowing on deep inspiration.

FIG. 2.

T.F.20 Before Atropir:

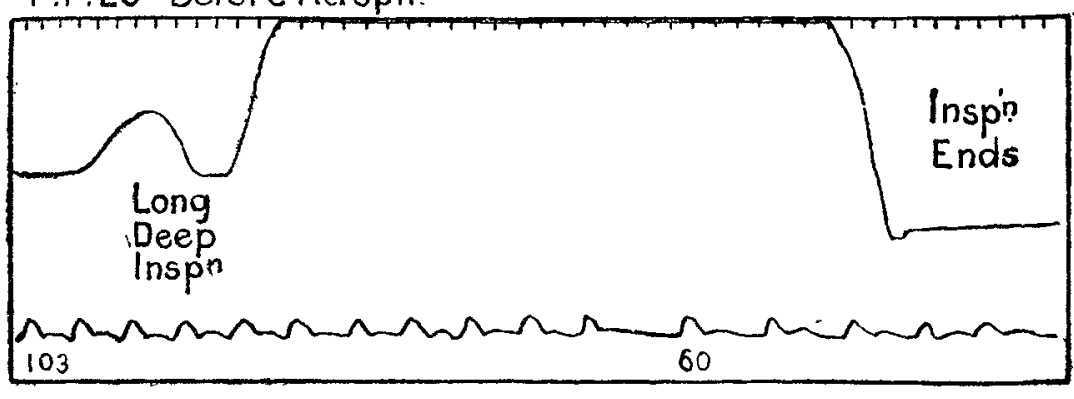

After Atropin $1 / 33$ grain (35 minutes after)

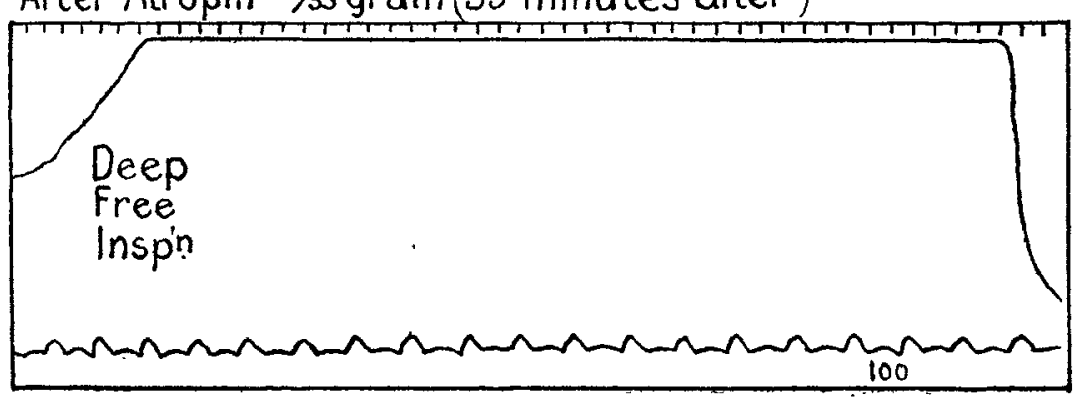

\section{$21 / 2$. Hours after Atropin}

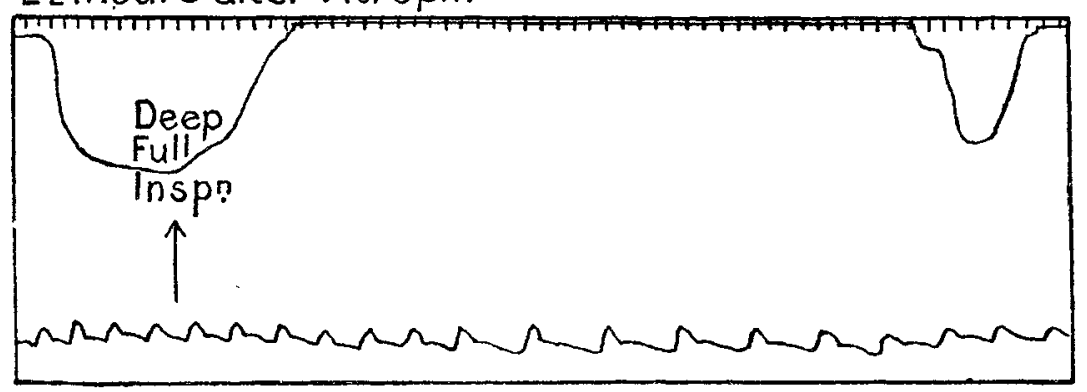

Slowing of pulse on deep inspiration, removed by atropine. vagus stimulation by deep inspiration had in this case :

1. Made it impossible for the compen. satory acceleration, the tachycardia, normally present to be kept up. Consequently the pulse slowed and acceleration was dropped, leaving only augmentation. There was a fall of blood pressure of $5-10 \mathrm{~mm}$. Hg systolic.

2. Finally placed the heart under such a strain that the contractile power was unable to cope with the over-filling, systole for diastole, so to speak. The organ was incapable, that is, of emptying itself or nearly emptying itself in a single systole. The mechanism of augmentation had, in fact, broken down.

Thus excessive vagus stimulation pro. duces the same effect as inhibition of the vagus by atropine. The reciprocal action of vagus and sympathetic can be dis. located in two ways-by "cutting out" the vagus, or by increasing the irritability of this nerve to an excessive degree. In both cases the only resort left to the heart is acceleration, which at once takes place.

\section{Types of Acceleration.}

There are thus three types of acceleration, all differing in their means of production :-

1. The type represented by the ordinary case of tachycardia, in which acceleration has been added to augmentation. The blood pressure is usually normal or slightly higher than normal-e.g. $140 \mathrm{~mm}$. Hg systolic.

2. The type occurring when augmentar tion is abolished by atropine. Blood pressure slightly less than normal.

3. The type occurring when augmentation is abolished by excessive irritability of the vagus. In this case the blood pressure falls sharply, to $80 \mathrm{~mm}$. $\mathrm{Hg}$ in many cases, and the patient feels faint. This type of acceleration is seen in the very severe cases of septic fevers, in pneumonia which is running to a fatal issue, and in other cases. It can also be

FIG. 3.

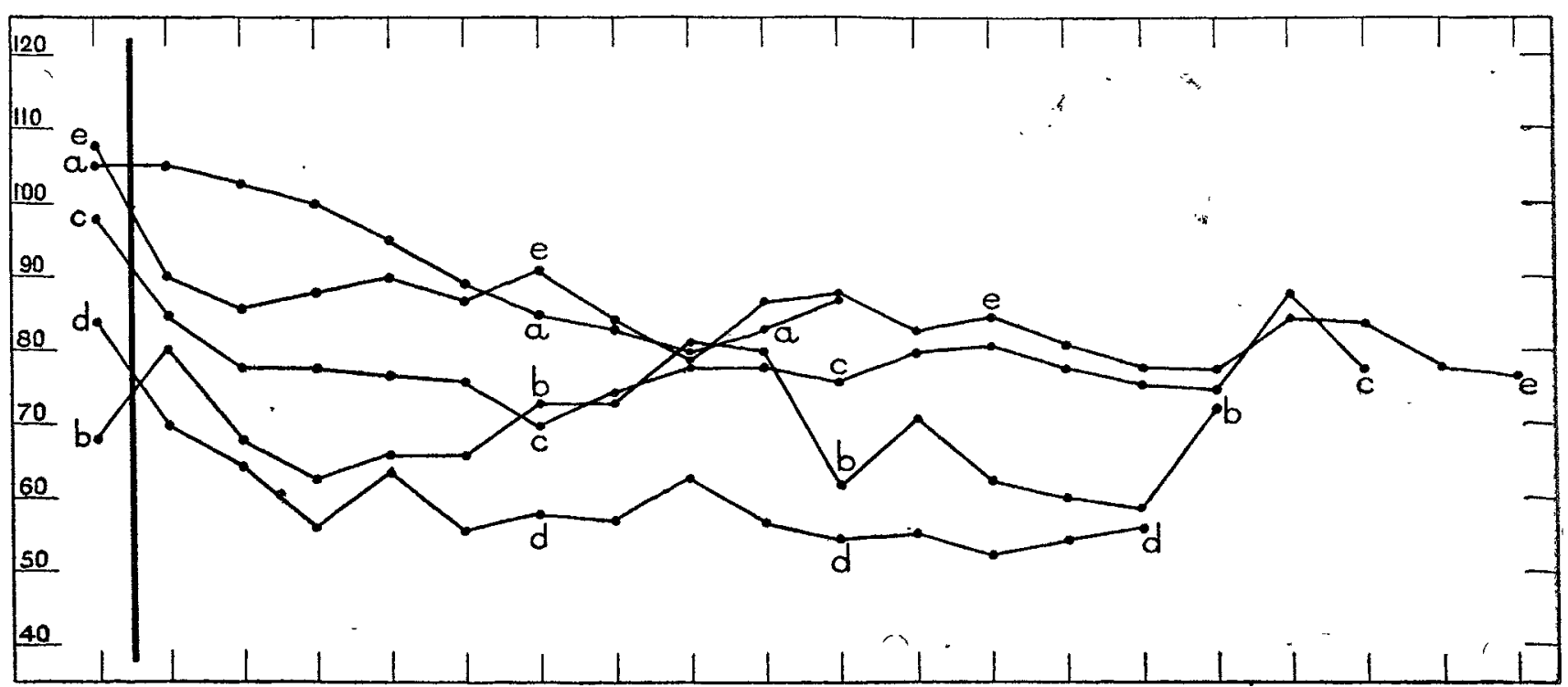

Graphic records of pulse-rate after a dose of $\frac{1}{20} \mathbf{g r}$. atropine intravenously. Tracings taken with polygrajh. The peryen ticular line shows point of injection. Note the fall in rate in all cases except $b$. 
FIG. 4.

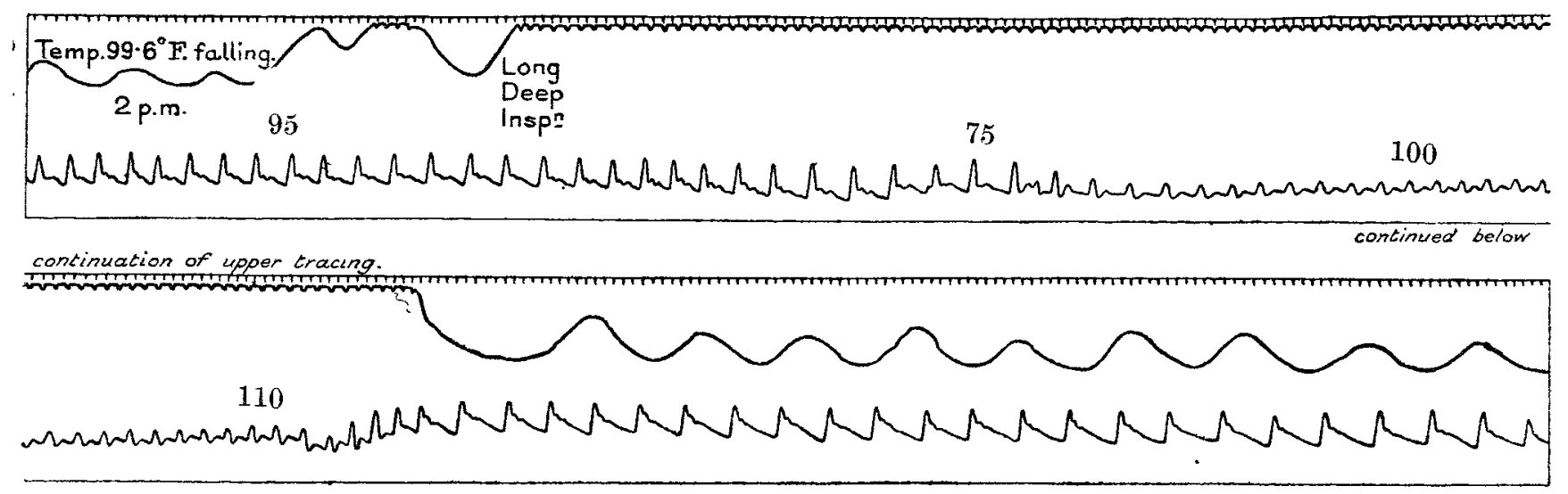

FIG. 5.

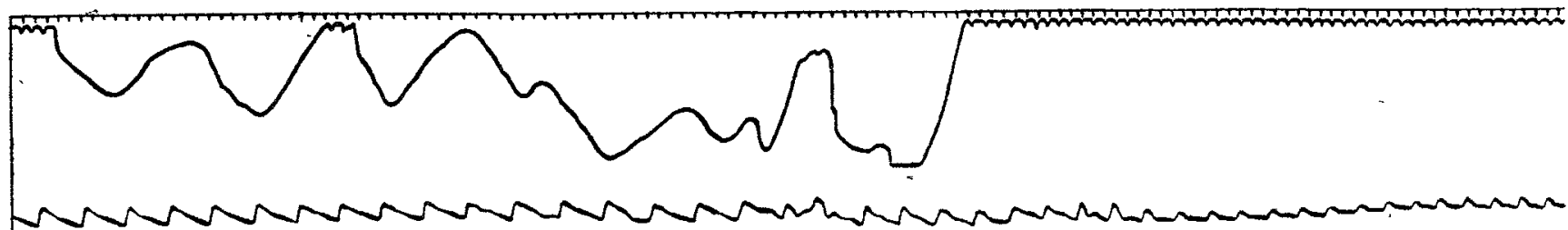
continued below

continuation of upper tracing.

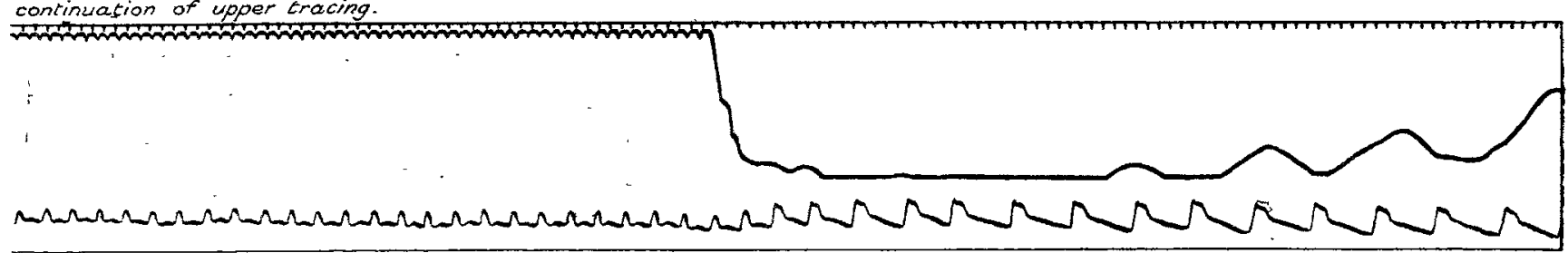

Sharp fall of blood pressure in case of trench fever with hyper-excitable vagus on deep inspiration.

FIG. 6.

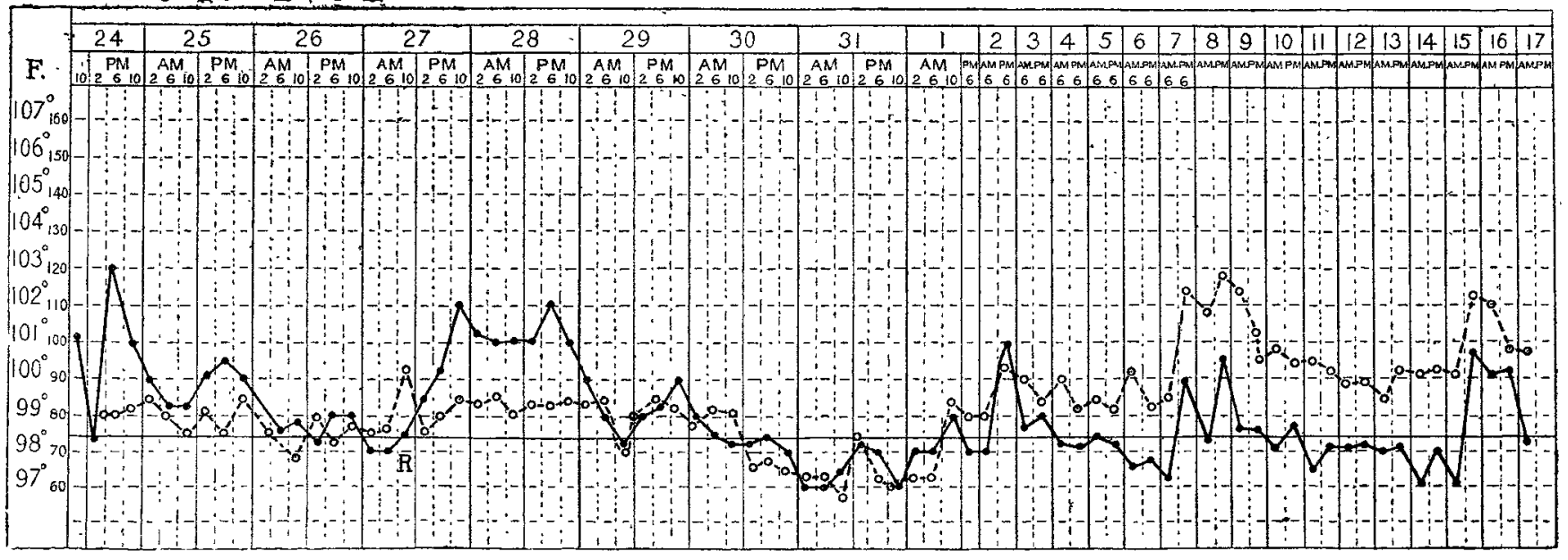

Temperature and pulse in trench fever.

produced, as has been shown, by vagus stimulation in some cases of tachycardia.

Tachycardia by this showing is a compensation to a hyper-excitable vagus. It remains to suggest that the vagus is so rendered hyper-excitable by the action of a toxin. In the accompanying chart from a case of trench ferer this can be seen. (Fig. 6.)

It will be observed that across the chart there is a gradual decline of the temperature curve and a gradual rise of the pulse curve. Moreover, the acceleration of the pulse towards the end of the chart increases with each slight rise of temperature. If the views enunciated are correct, the explanation is that while at first the toxin was powerful enough to render the vagus unduly excitable, and so to prevent acceleration towards the later stage of the illness, the toxin had lost power and acceleration could occur. In point of fact, the blood pressure, which had been subnormal at the beginning, became normal later when the compensatory acceleration was established. The same tendency to the establishment of tachycardia as fever declines can be observed in many influenza charts and in other diseases. When tachycardia is established the patient is better in his strength than before this takes place. Conclusions.

1. Tachycardia in a man who is up and about is compensatory in character, the addition of acceleration to augmentation.

2. It points to the presence of a mild infection, and can be removed only by removing the infection.

3 . There is evidence that the action of the toxin is directly on the cardiac vagus. 